\title{
On the generation mechanisms of short-scale unbalanced modes in rotating two-layer flows with vertical shear
}

\author{
By PAUL D. WILLIAMS ${ }^{1} \uparrow$, THOMAS W. N. HAINE \\ AND PETER L. REA D \\ ${ }^{1}$ Atmospheric, Oceanic and Planetary Physics, Clarendon Laboratory, Parks Road, \\ Oxford OX1 3PU, UK \\ ${ }^{2}$ Department of Earth and Planetary Sciences, 329 Olin Hall, 34th and North Charles Streets, \\ Johns Hopkins University, Baltimore, MD 21218, USA
}

(Received 4 May 2004 and in revised form 20 October 2004)

We report on the results of a laboratory investigation using a rotating two-layer annulus experiment, which exhibits both large-scale vortical modes and short-scale divergent modes. A sophisticated visualization method allows us to observe the flow at very high spatial and temporal resolution. The balanced long-wavelength modes appear only when the Froude number is supercritical (i.e. $F>F_{\text {critical }} \equiv \pi^{2} / 2$ ), and are therefore consistent with generation by a baroclinic instability. The unbalanced shortwavelength modes appear locally in every single baroclinically unstable flow, providing perhaps the first direct experimental evidence that all evolving vortical flows will tend to emit freely propagating inertia-gravity waves. The short-wavelength modes also appear in certain baroclinically stable flows.

We infer the generation mechanisms of the short-scale waves, both for the baroclinically unstable case in which they co-exist with a large-scale wave, and for the baroclinically stable case in which they exist alone. The two possible mechanisms considered are spontaneous adjustment of the large-scale flow, and Kelvin-Helmholtz shear instability. Short modes in the baroclinically stable regime are generated only when the Richardson number is subcritical (i.e. $R i<R i_{\text {critical }} \equiv 1$ ), and are therefore consistent with generation by a Kelvin-Helmholtz instability. We calculate five indicators of short-wave generation in the baroclinically unstable regime, using data from a quasi-geostrophic numerical model of the annulus. There is excellent agreement between the spatial locations of short-wave emission observed in the laboratory, and regions in which the model Lighthill/Ford inertia-gravity wave source term is large. We infer that the short waves in the baroclinically unstable fluid are freely propagating inertia-gravity waves generated by spontaneous adjustment of the large-scale flow.

\section{Introduction}

The Navier-Stokes equations for a rotating, incompressible and stably stratified fluid flow generally permit linear normal mode solutions which fall into two distinct categories, as first identified by Margules (1893). The balanced, or vortical modes have a relative vorticity which is large compared to their horizontal velocity divergence.

$\dagger$ Present address: Centre for Global Atmospheric Modelling, Department of Meteorology, University of Reading, PO Box 243, Earley Gate, Reading RG6 6BB, UK. 
These modes are comparatively slowly evolving and large in horizontal wavelength. In contrast, the unbalanced, or divergent modes have a horizontal velocity divergence which is large compared to their relative vorticity. These modes propagate relatively quickly and are short in horizontal wavelength. Important comparisons between the characteristic properties of these two normal mode classes have been presented by Hough (1898) and Longuet-Higgins (1968).

The detailed form taken by eigensolutions in both categories depends on the background flow upon which they are superimposed. For a background state with a sufficiently small vertical shear in horizontal velocity, the vortical and divergent modes are manifest as Rossby waves and inertia-gravity waves, respectively, both of which may theoretically propagate with constant amplitude in the absence of dissipation. If the vertical shear in horizontal velocity is sufficiently large that the Richardson number is subcritical (i.e. $R i<R i_{\text {critical }} \sim 1$ ), then small-amplitude divergent modes are able to extract energy from the background flow and grow in amplitude owing to a rotationally modified Kelvin-Helmholtz instability (e.g. Chandrasekhar 1961). Similarly, if the Froude number is supercritical (i.e. $F>F_{\text {critical }} \equiv \pi^{2} / 2$ ), then smallamplitude vortical modes are able to extract energy from the background flow and grow owing to a baroclinic instability. These two hydrodynamic instabilities are important sources of divergent and vortical waves, respectively, in rapidly rotating flows with vertical shear.

Outside the regime in which Kelvin-Helmholtz instability is exhibited, the existence of a divergent mode eigensolution in the equations of motion is simply an indication that inertia-gravity wave motions are supported by the fluid. It is not necessarily a guarantee that inertia-gravity waves will be generated, however, since generation requires an external mechanism in a flow without instability. Possible inertia-gravity wave generation mechanisms include interactions between the flow and a physical obstruction, as investigated in the Lighthill (1978) ship-wake problem, for example. Topography is a special case of a physical obstruction, and is an important source of atmospheric gravity waves in mountainous regions (e.g. Hines 1988). A further potential inertia-gravity wave source is the excess energy associated with the adjustment of the large-scale flow. This includes (but is not limited to) the classical geostrophic adjustment process first described by Rossby (1938), in which an unbalanced vortical flow self-adjusts, in order to tend to establish geostrophic balance, by emitting transient inertia-gravity waves. Ford (1994) showed that balanced (to first order) vortical flows can also generate inertia-gravity waves by a generalized adjustment process, and he used the shallow-water equations to derive a source term for generation due to this mechanism. Ford, McIntyre \& Norton (2000) prefer the term spontaneous adjustment to geostrophic adjustment, since the process need not necessarily take the flow toward geostrophic balance.

Perhaps because of their meteorological significance, the dominant mechanisms by which vortical modes are generated and sustained have been extensively studied (see, for example, the review article by Klein 1990). The generation of divergent modes has received significantly less attention, despite their ubiquitous presence in atmospheric obervations (e.g. Eckermann \& Vincent 1993; Sato 1994; Dalin et al. 2004). As a result, it seems unclear whether the dominant source of divergent modes in the free atmosphere (i.e. away from topography) is a local Kelvin-Helmholtz shear instability, or is instead radiation of excess energy associated with spontaneous adjustment of the larger-scale flow. Generation by both processes has been investigated (the former by Fritts 1982, 1984; the latter by Fritts \& Luo 1992 and Luo \& Fritts 1993), but firm conclusions about which mechanism dominates have not yet been reached. This 
long-standing uncertainty clearly must be resolved, since it seems unlikely that we will be able to successfully predict regions of intense short-scale wave activity in fluids (including those of geophysical relevance) until we have at least established which is the dominant mechanism by which the waves are generated and maintained.

In this paper, we describe laboratory, semi-analytic and numerical investigations of the sources of short-wave emission in the rotating two-layer annulus. Laboratory experiments are an important research tool in this case, since the system is under the complete control of the experimenter; global high-resolution measurements can be taken systematically; and experiments can be repeated as many times as required. It would be difficult, if not impossible, to enjoy these benefits if short-wave emission were investigated directly in the atmosphere rather than in the laboratory. Of course, the dynamical and geometrical similarity between the fluid in a rotating laboratory experiment and the atmosphere on a rotating planet is not exact, but we can choose a reasonable correspondence by matching the laboratory and atmosphere regimes as closely as possible.

Rotating two-layer laboratory systems such as that employed in the present study have been used for decades to investigate baroclinic instability and vortical mode interactions (e.g. Hart 1972; King 1979; Bradford, Berman \& Lundgren 1981; Hart 1985). The spontaneous emission of gravity waves by interacting vortex dipoles in a stratified fluid has been studied in the laboratory by Afanasyev (2003). The possibility of studying inertia-gravity wave generation by an evolving large-scale flow in the laboratory was first reported by Lovegrove, Read \& Richards (1999). Previously, Read (1992a) had reported observations of fast oscillations in the temperature field of a rotating thermal annulus experiment. The oscillations appeared to be inertiagravity waves, but it is possible that they were generated by the thermocouple probes which made the temperature measurements, acting as physical obstructions to the flow. This apparatus was therefore not ideally suited for studying the generation of inertia-gravity waves by the internal dynamics of the fluid. To overcome this problem, Lovegrove et al. (1999) used a novel flow-visualization technique, first described by Hart \& Kittelman (1986), in an isothermal annulus with two discrete layers. The method is non-invasive, ruling out the possibility of an interaction between the flow and the measurement device. This is the visualization technique which we use in the present study, since it allows us to conclude with confidence that any observed short-scale waves originate solely from the internal dynamics of the fluid.

The layout of this paper is as follows. In $\S 2$, we describe the laboratory apparatus which we use in this study. We describe experiments we have performed in order to determine the conditions under which short unbalanced waves are generated, and find that short-wave emission occurs both in the baroclinically stable and baroclinically unstable regimes. This means that the short waves can appear both alone, and superimposed on a large-scale vortical mode with which they co-exist. In the remainder of the paper, we investigate the generation mechanisms which give rise to the shortscale waves in both cases. Taking the baroclinically stable regime first, in $\S 3$, we use a semi-analytical approach to evaluate the Richardson number at a wide range of parameter values. We find that there is good agreement between regions of subcritical Richardson number and regions of short-scale wave generation (except perhaps near the curve of marginal baroclinic instability), and we conclude that a Kelvin-Helmholtz instability is the generation mechanism in this case. Then, in $\S 4$, we employ a numerical model to calculate various indicators of short-wave generation in the baroclinically unstable regime. By establishing which indicator best predicts the spatial locations of the short-wave generation observed in the laboratory, we 


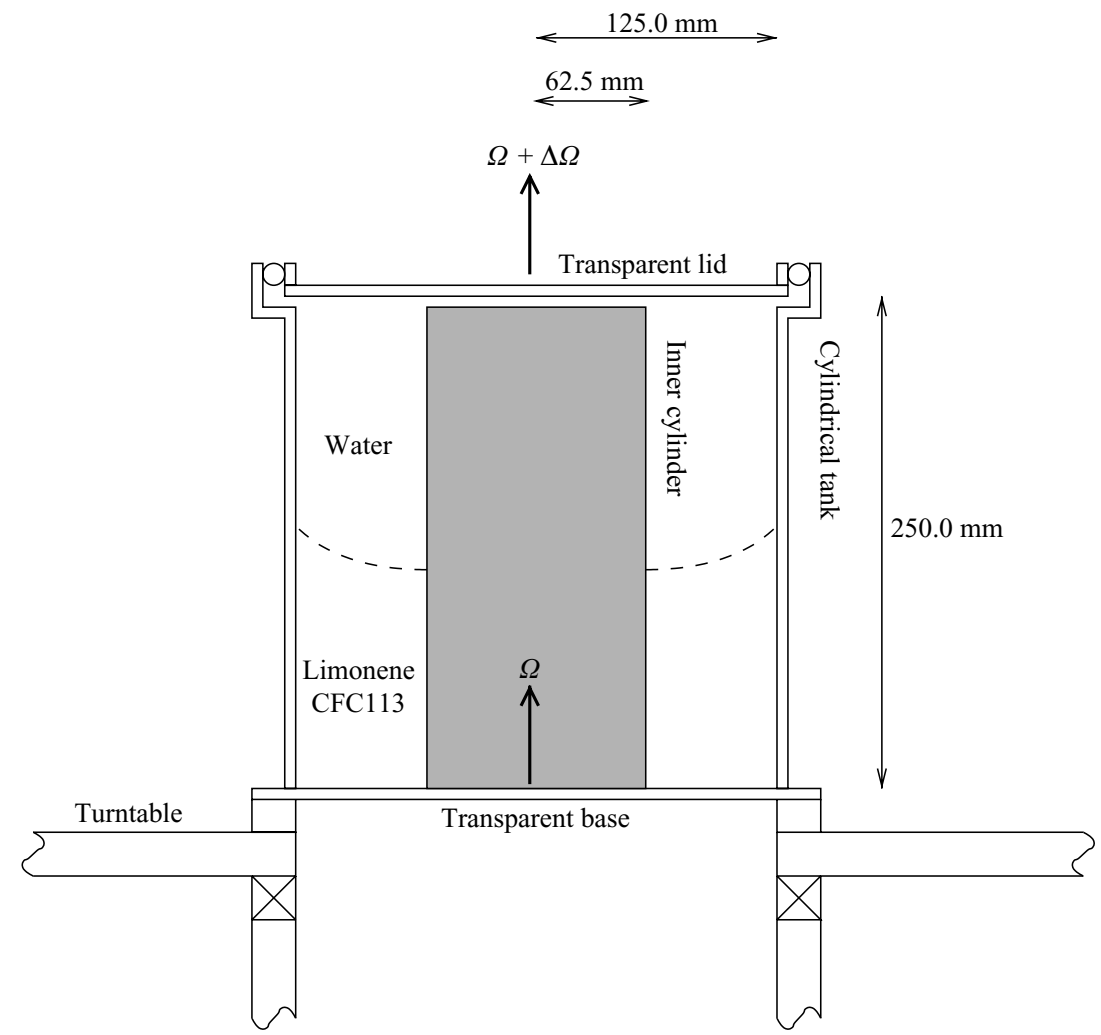

FIGURE 1. Schematic cross-section through the two-layer annulus apparatus used in this study, showing the principal components described in the text. The interface between the two liquids, which have equal volumes, is shown as a dashed line. The angular velocities of the turntable and lid, in the laboratory frame, are indicated. A bright white source light illuminates the apparatus from below (not shown). After propagating vertically upwards through the fluids, the light is received by a colour video camera, which is mounted on the rotation axis around $2 \mathrm{~m}$ above the turntable (also not shown).

conclude that the spontaneous adjustment of the large-scale flow is most likely to be the mechanism responsible in this case, in contrast with the baroclinically stable case. Finally, in $\S 5$, we discuss the results and their implications, and suggest possible avenues for future work.

\section{Details of the laboratory investigation}

In this section, we describe the laboratory apparatus employed in this study, and give the results of the present series of experiments.

\subsection{The rotating two-layer annulus apparatus}

For the laboratory investigation, we use the same apparatus as that used by Lovegrove et al. (1999, 2000). A brief description is given here, and the interested reader is referred to those papers for further details.

A vertical cross-section through the apparatus is shown schematically in figure 1. The annulus has an inner vertical sidewall of radius $62.5 \mathrm{~mm}$, an outer vertical sidewall of radius $125.0 \mathrm{~mm}$, and a total depth of $250.0 \mathrm{~mm}$. The radial width of the annulus gap is therefore $L=62.5 \mathrm{~mm}$, and the two immiscible liquid layers have 


\begin{tabular}{|c|c|c|c|c|c|c|c|c|}
\hline & & & & $\begin{array}{l}\text { er layer } \\
\text { ater) }\end{array}$ & & $\begin{array}{r}\text { Lon } \\
\text { (limonene }\end{array}$ & $\begin{array}{l}\text { ayer } \\
\text { CFC }\end{array}$ & \\
\hline $\begin{array}{l}\text { Density, } \rho(\mathrm{kg} \mathrm{m} \\
\text { Kinematic visco } \\
\text { Optical activity }\end{array}$ & $\begin{array}{l}v\left(10^{-}\right. \\
59 \mu \mathrm{m}\end{array}$ & $\begin{array}{l}\left.2 \mathrm{~s}^{-1}\right) \\
\left.\mathrm{g} \cdot \mathrm{m}^{-1}\right)\end{array}$ & & $\begin{array}{l}7 \pm 1 \\
\pm 0.02 \\
0\end{array}$ & & $\begin{array}{r}10 \\
1.08 \\
77\end{array}$ & $\begin{array}{l}-1 \\
.02 \\
10\end{array}$ & \\
\hline TABLE 1. Selected & ical & $\begin{array}{l}\text { erties } \\
\text { ten }\end{array}$ & $\begin{array}{l}\text { the ty } \\
\text { eratur }\end{array}$ & $\begin{array}{l}\text { vorking } \\
\left(0^{\circ} \mathrm{C}\right)\end{array}$ & uids & at the am & nt la & ory \\
\hline $\begin{array}{l}\text { Experiment number } \\
\Delta \Omega\left(\mathrm{rad} \mathrm{s}^{-1}\right)\end{array}$ & $\begin{array}{c}1 \\
0.62\end{array}$ & $\begin{array}{c}2 \\
0.69\end{array}$ & $\begin{array}{c}3 \\
0.77\end{array}$ & $\begin{array}{c}4 \\
0.85\end{array}$ & $\begin{array}{c}5 \\
0.95\end{array}$ & $\begin{array}{c}6 \\
1.06\end{array}$ & $\begin{array}{c}7 \\
1.19\end{array}$ & $\begin{array}{c}8 \\
1.31\end{array}$ \\
\hline $\begin{array}{c}\text { Experiment number } \\
\Delta \Omega\left(\mathrm{rad} \mathrm{s}^{-1}\right)\end{array}$ & $\begin{array}{c}9 \\
1.46\end{array}$ & $\begin{array}{c}10 \\
1.61\end{array}$ & $\begin{array}{c}11 \\
1.80\end{array}$ & $\begin{array}{c}12 \\
2.03\end{array}$ & $\begin{array}{c}13 \\
2.24\end{array}$ & $\begin{array}{c}14 \\
2.51\end{array}$ & $\begin{array}{c}15 \\
2.73\end{array}$ & $\begin{array}{c}16 \\
3.14\end{array}$ \\
\hline
\end{tabular}

TABLE 2. The differential lid rotation, $\Delta \Omega$, used in each of the present experiments.

equal resting depths of $H=125.0 \mathrm{~mm}$. The base and lid are both horizontal and flat, and the annulus is mounted on a turntable. The base and sidewalls rotate under computer control with angular velocity $\Omega$ about the axis of symmetry, and the lid (in contact with the upper fluid layer) rotates under computer control relative to the base and sidewalls with angular velocity $\Delta \Omega$, so that the angular velocity of the lid in the laboratory frame is $\Omega+\Delta \Omega$. This differential rotation provides a velocity shear which is concentrated at the Ekman layers at the lid, base and fluid interface. For all of the experiments described herein, we use $\Omega>0$ and $\Delta \Omega>0$. This means that, when viewed from above, the turntable rotation is anticlockwise in the laboratory frame, and the lid rotation is anticlockwise in the turntable frame, with a super-rotation of the lid and fluids relative to the turntable.

For the upper layer we use water, and for the lower layer we use a mixture of limonene and CFC-113. These two liquids are both practically insoluble in water, and are mixed in such proportions that the composite density is slightly greater than that of water. A summary of some of the physical properties of the liquids is given in table 1. Importantly, the lower-layer liquid has a large optical activity, owing to the limonene. This means that, when we illuminate the system from below with white light, and view it through crossed polaroids from above using a video camera, there is a correlation between the colour registered by the camera and the depth of the lower layer. This is the flow-visualization method first proposed by Hart \& Kittelman (1986), offering a convenient and non-invasive method for visualizing the two-dimensional shape of the interface at high resolution in both the horizontal coordinate $(0.5 \mathrm{~mm})$ and time $(0.04 \mathrm{~s})$. The natural interfacial tension between the two liquids is quite large $\left(2.9 \times 10^{-2} \mathrm{~N} \mathrm{~m}^{-1}\right)$, and so for all of the experiments described in this paper, we add a surfactant to the fluids, which reduces it to $5.3 \times 10^{-3} \mathrm{~N} \mathrm{~m}^{-1}$.

\subsection{Results of the laboratory experiments}

We have conducted a series of 16 experiments using the rotating annulus shown in figure 1, with the aim of categorizing the presence of vortical and/or divergent modes at a wide range of parameter values. The differential lid rotation, $\Delta \Omega$, was held constant during each experiment, at the values shown in table 2 . In order to achieve a continuous scan of parameter space, each experiment (which lasted three hours - much longer than the spin-up time of around one minute) had a turntable 
(a)

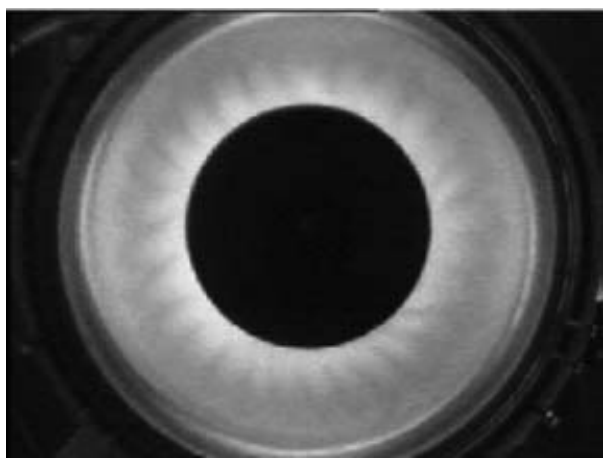

(c)

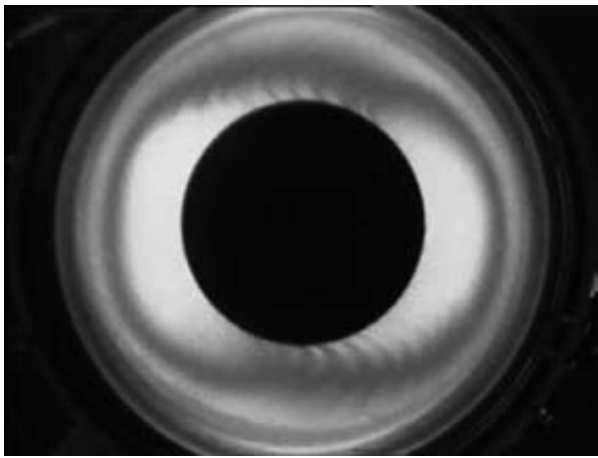

(b)

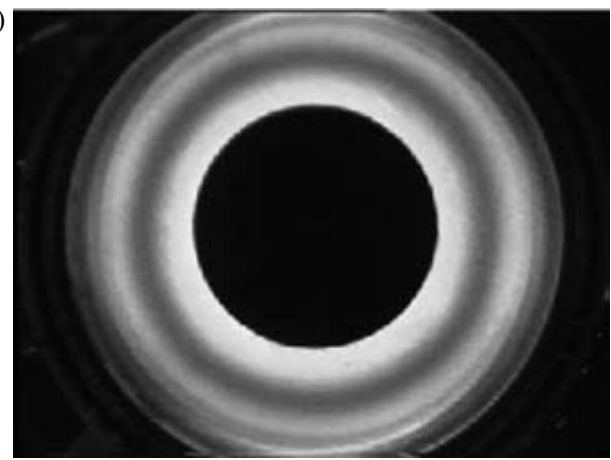

$(d)$

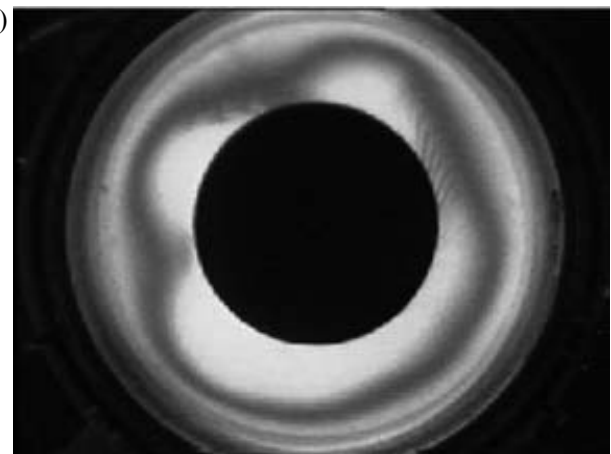

FIGURE 2. Monochrome versions of colour images recorded by the video camera which views the laboratory annulus in figure 1 from above. In all four cases the differential lid rotation, $\Delta \Omega$, is $0.95 \mathrm{rad} \mathrm{s}^{-1}$. The background turntable rotation, $\Omega$, is slowly and continuously increased between $(a)$ and $(d)$, taking values of $(a) 0.5 \mathrm{rad} \mathrm{s}^{-1}, K H$ regime, $(b) 1.4 \mathrm{rad} \mathrm{s}^{-1}, A X$ regime, (c) $2.1 \mathrm{rad} \mathrm{s}^{-1}, M R W$ regime and (d) $3.3 \mathrm{rad} \mathrm{s}^{-1}, M I W$ regime.

angular velocity, $\Omega$, which gradually increased in time at a constant rate of $\mathrm{d} \Omega / \mathrm{d} t=$ $4.0 \times 10^{-4} \mathrm{rad} \mathrm{s}^{-2}$, starting from rest. This meant that the turntable rotation had reached $\Omega=4.3 \mathrm{rad} \mathrm{s}^{-1}$ by the end of each experiment, which is around the largest rate that can be used without the fluid interface intersecting the annulus lid and/or base. Two-layer systems with unequal viscosities may be susceptible to instabilities at the interface during spin-up (Berman, Bradford \& Lundgren 1978). Such instabilities are never observed to occur in the present experiments, since the viscosities are approximately equal (table 1) and the angular acceleration is sufficiently small that the fluids may be regarded as being fully spun-up at all times.

During a typical experimental run, the flow types observed fell into the following four distinct classes, when categorized according to the presence and absence of largeand short-scale perturbations to the interface height. The flow types are illustrated in figure 2 by images from experiment number 5 , which had $\Delta \Omega=0.95 \mathrm{rad} \mathrm{s}^{-1}$. These images are representative of the flow types observed in all 16 experiments.

(i) Kelvin-Helmholtz regime, $\mathrm{KH}$. Absence of large-scale waves with global presence of short-scale waves, as shown in figure $2(a)$.

(ii) Axisymmetric regime, $A X$. Absence of both large-scale and short-scale waves, as shown in figure $2(b)$.

(iii) Mixed regular wave regime, $M R W$. Presence of regular large-scale waves together with local presence of short-scale waves, as shown in figure 2(c). 
(iv) Mixed irregular wave regime, MIW. Presence of irregular large-scale waves together with local presence of short-scale waves, as shown in figure $2(d)$.

In general, during a three hour run, the order in which the flow types are encountered is $A X \rightarrow K H \rightarrow A X \rightarrow M R W \rightarrow M I W$, so that there are four major transitions in total (cf. wavenumber transitions within the $M R W$ and $M I W$ regimes, which are not studied here). The $K H$ regime is so-called in anticipation of evidence to be presented in $\S 3$ that the short-scale waves in this regime are, indeed, waves generated by a Kelvin-Helmholtz instability, rather than freely propagating inertia-gravity waves. As is the case for geophysical flows, there is a distinct spectral gap between the characteristic spatial and temporal scales of the two modes. For practical purposes, waves with azimuthal wavenumbers below five were regarded as large-scale waves, and all others as short-scale waves, though typically the spectral gap was much larger than that permitted by this definition, as suggested by figure 2 . We have developed a method for inferring interface heights from the colour video camera images (Williams, Read \& Haine 2004b), and thereby established that the amplitudes of the largewavelength modes typically exceed those of the short-wavelength modes by a factor of around 10 (Williams, Read \& Haine 2003).

The unbalanced short-scale modes have different characteristic properties when they appear in the $M R W$ and $M I W$ regimes rather than in the $K H$ regime. This can be seen by comparing figures $2(c)$ and $2(d)$ with $2(a)$, for example. In the $M R W$ and $M I W$ regimes, the short waves are generally smaller in wavelength, and are azimuthally confined, unlike in the $K H$ regime. This suggests that different generation mechanisms may be responsible in the two cases, a possibility which we investigate in $\S \S 3$ and 4 . The characteristic chevron-shaped structure of the short waves in the $M R W$ and $M I W$ regimes closely resembles that of the inertia-gravity waves generated by geostrophic adjustment of the tropospheric jetstream, in the high-resolution numerical simulations of O'Sullivan \& Dunkerton (1995).

For the purposes of constructing an experimental regime diagram, we employ two dimensionless parameters, the Froude number $(F)$ and dissipation parameter $(d)$, defined by

$$
F=\frac{4 \Omega^{2} L^{2}}{g^{\prime} H}
$$

and

$$
d=\frac{\sqrt{\bar{\nu} \Omega}}{H \Delta \Omega}
$$

where $g^{\prime}=g \Delta \rho / \bar{\rho}=5.9 \mathrm{~cm} \mathrm{~s}^{-2}$ is the reduced gravity, $\bar{v}=1.18 \times 10^{-6} \mathrm{~m}^{2} \mathrm{~s}^{-1}$ is the mean kinematic viscosity, and $L$ and $H$ are the annulus dimensions, as defined in $\S 2.1$.

For each of the 16 experiments, we can evaluate the rotation rates, $\Delta \Omega$ and $\Omega$, for each transition between the four flow types defined above. This allows the Froude number and dissipation parameter corresponding to each transition to be calculated using (2.1) and (2.2). The results thereby obtained are summarized in the regime diagram of figure 3. Contours of constant Rossby number, $R o=\Delta \Omega /(2 \Omega)$, are shown in the figure for reference, showing that we have investigated flows in which this parameter varies by three orders of magnitude, encompassing the geostrophic $(R o<1)$ and ageostrophic $(R o>1)$ regimes. The full Froude number span was not always achieved, as some of the higher-numbered experiments were terminated before the full three hours had elapsed. This was because the fluid interface had become so steep that it began to intersect the annulus lid. 


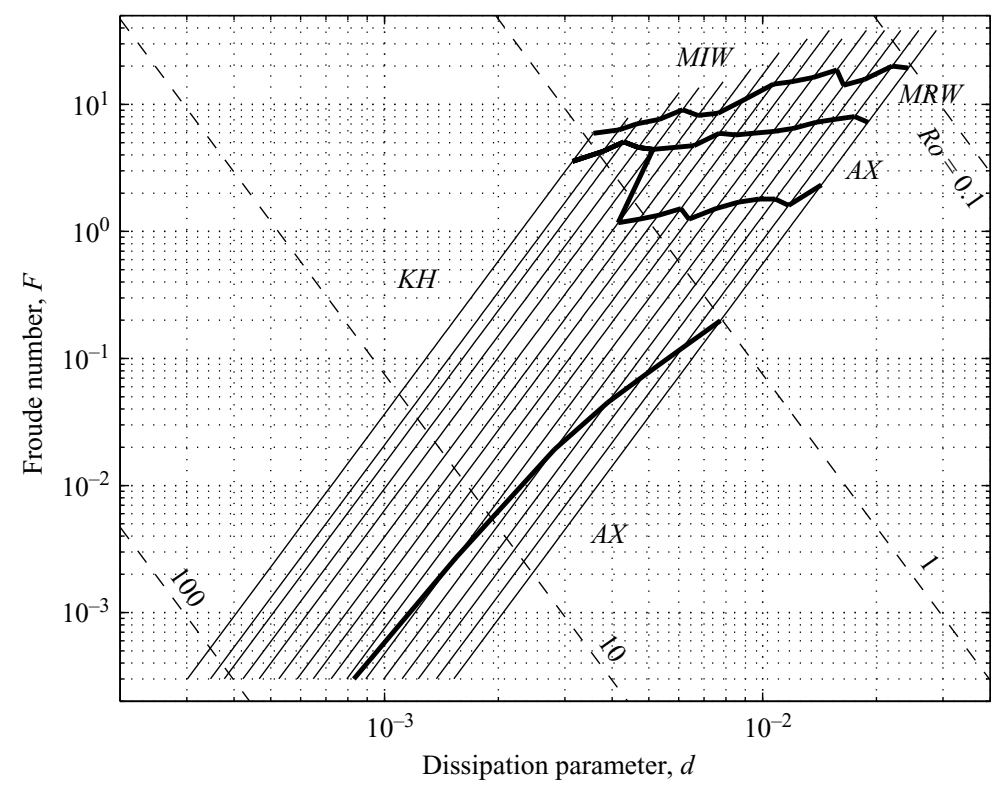

FIGURE 3. The experimental regime diagram, in the plane defined by the Froude number and dissipation parameter. Each of the 16 thin straight lines corresponds to one three-hour experiment, with experiment 1 furthest to the right and experiment 16 furthest to the left (see table 2). The 16 regime scans are in the direction from small to large Froude number. Contours of constant Rossby number, $R o$, are over-plotted (dashed lines). The boundaries between the four regimes discussed in the text $(A X, K H, M I W$ and $M R W)$ are delineated by thick, solid lines.

The presence of large-scale waves in the $M R W$ and $M I W$ regimes is due to a baroclinic instability (Hart 1972), and there is agreement between the locations of the experimental and theoretical marginal baroclinic instability curves. The Phillips model (e.g. Pedlosky 1987) predicts a Froude number for this curve of $F_{\text {critical }}=\pi^{2} / 2 \approx 4.9$, independent of the vertical shear (i.e. the dissipation parameter). This is in reasonable agreement with the Froude numbers for the transitions to the $M R W$ regime in figure 3. The experimental critical Froude number varies from around 4 to 7 as the dissipation parameter is increased, implying a weak dependence of the marginal baroclinic instability curve on shear. This effect is not captured by the standard Phillips model, but would seem to be in accord with the frictional Phillips theory. The frictional effects of the Ekman layers have a stabilizing influence (\$7.12 of Pedlosky 1987), with the implication that there is a minimum shear required for instability. As the dissipation increases, the minimum Froude number required to overcome the stabilizing effects increases, in accord with the marginal baroclinic instability curve of our regime diagram. The experiments of Griffiths \& Linden (1981) also appear to reach marginal instability when such a critical shear is attained. A similar theory is developed for cylindrical geometry by Hart (1972). The $M R W \rightarrow M I W$ transition in figure 3 represents the point at which the forcing becomes so strong that regular vortical motions are no longer dynamically permitted, and are replaced by irregular chaotic motions.

The interpretation of the shapes of the $A X \rightarrow K H$ and $K H \rightarrow A X$ transition curves is not as obvious as for the $A X / K H \rightarrow M R W$ and $M R W \rightarrow M I W$ curves. As the Froude number is increased at relatively high values of the dissipation parameter, 
the $A X \rightarrow K H$ transition is only temporary, being followed shortly after by the reverse transition. In $\S 3$, we infer the generation mechanism of the short-scale waves in the baroclinically stable fluid, from the shapes of the $A X \leftrightarrow K H$ regime boundaries.

Three further series of 16 experiments have been carried out: one with retrograde lid rotation $(\Delta \Omega<0)$; one with decreasing, rather than increasing turntable rotation $(\mathrm{d} \Omega / \mathrm{d} t<0)$; and one with the inner cylinder displaced horizontally by around $2 \mathrm{~cm}$ to give an eccentric annular geometry. These additional experiments are discussed no further here, except to state that in each different configuration, each of the four regimes discussed above was clearly identified, demonstrating that the wavegeneration mechanisms are reasonably robust.

An important observation, from the series of experiments described above, is that localized short-scale waves develop during every single baroclinically unstable flow (the $M R W$ and MIR regimes). This agrees with the hypothesis of Ford et al. (2000) that, with few if any exceptions, all unsteady vortical flows will tend to emit freely propagating inertia-gravity waves, implying the non-existence of a strict slow manifold. In the present study, a preliminary series of laboratory experiments (without added surfactant) did not exhibit short-scale waves in the $M R W$ and $M I R$ regimes, but this seems to be due to suppression of their generation by the large interfacial tension in that case. Lovegrove et al. (2000) have also reported the generation of shortscale waves in the baroclinically unstable two-layer annulus, but only in restricted circumstances, namely when the amplitude of the large-scale mode vacillates periodically in time. We speculate that higher interfacial tension and/or lower visualization resolution may have prevented the generation and/or observation of more ubiquitous short-scale waves in those experiments. An important outcome of the present series of experiments, therefore, is that we have obtained corroborative laboratory evidence to support the inertia-gravity wave generation theory of Ford et al. (2000). We investigate the applicability of this theory to the present laboratory experiments in more detail in $\S 4$, when we examine the generation of short-scale waves by the large-scale flow.

\section{Unbalanced modes in the baroclinically stable fluid}

We now show that the short waves in the $K H$ regime, i.e. those present in figure $2(a)$, are Kelvin-Helmholtz modes generated by a shear instability, as anticipated by the label. Kelvin-Helmholtz modes are expected to be generated wherever and whenever the vertical shear in horizontal velocity exceeds a particular value dependent on the stratification. This criterion is expressed in non-dimensional terms as the Richardson number dropping below some critical value, usually taken to be $1 / 4$ or 1 depending upon the exact definition of the Richardson number (Acheson 1990).

The gradient Richardson number for a one-dimensional continuously stratified flow is defined by

$$
R i_{\mathrm{g}}=\frac{-(g / \rho)(\partial \rho / \partial z)}{(\partial u / \partial z)^{2}},
$$

where $\rho(z)$ and $u(z)$ are the density and horizontal velocity profiles, respectively. For an axisymmetric two-layer annulus flow, with both layers in solid-body rotation with rates $\Delta \Omega_{1}$ (upper layer) and $\Delta \Omega_{2}$ (lower layer) relative to the turntable, the bulk Richardson number at radius $r$ is

$$
R i_{\mathrm{b}}=\frac{2 g^{\prime} \sqrt{\bar{v} / \Omega}}{r^{2}\left(\Delta \Omega_{1}-\Delta \Omega_{2}\right)^{2}} .
$$


A vertical average has been taken over the interfacial Ekman (1905) layers, where it is assumed that the shear is concentrated, as follows from the Taylor-Proudman theorem (e.g. Acheson 1990). There are two interfacial Ekman layers, one directly above and one directly below the interface, and each has width $\sqrt{\bar{v} / \Omega}$ (e.g. Read 1992b).

The Richardson number criterion stated above applies to non-rotating systems. The applicability of non-rotating Kelvin-Helmholtz instability theory to rotating systems has been investigated by James (1977). He derives an implicit fourth-order polynomial dispersion relation for waves in a rotating two-layer channel, and solves it numerically to plot Kelvin-Helmholtz growth rate curves for both the rotating and non-rotating cases. A comparison of the curves leads him to conclude that 'the Kelvin-Helmholtz instability is but little affected by rotation ... broadly, this [rotating case] instability is adequately described by the non-rotating theory'. This statement holds because the short-scale waves evolve on time scales of much less than the rotation period. We conclude that we may apply the non-rotating criterion to the present system.

We now evaluate the annulus Richardson number, given by (3.2), at the rotation rates used in the laboratory experiments, to determine whether there is agreement between the incidence of laboratory short waves and subcritical Richardson number. In order to achieve this, we must determine the layer rotation rates, $\Delta \Omega_{1}$ and $\Delta \Omega_{2}$, in terms of $\Omega$ and $\Delta \Omega$. We have developed a semi-analytical method for this purpose, based on a layerwise balance of the torques due to the thin viscous boundary layers. Acting on the inviscid interiors of both the upper and lower fluids, there is a positive (prograde) torque due to the shear across the upper Ekman layer, and there are negative (retrograde) torques due to the shears across the lower Ekman layer and the Stewartson (1957) layers at the outer and inner vertical sidewalls. By requiring the torques in each layer to balance in equilibrium (otherwise there would be a nonzero angular acceleration), we obtain two coupled nonlinear equations which can be solved iteratively for $\Delta \Omega_{1}$ and $\Delta \Omega_{2}$. The full details of the calculation are described in Williams et al. (2004b).

The results of this calculation for each combination of 7 different values of $\Delta \Omega$ and 16 different values of $\Omega$, spanning approximately the same ranges as achieved in the laboratory experiments, are shown in figure $4(a)$. For combinations where both $\Omega$ and $\Delta \Omega$ are large, the iterative solution fails to converge, and so these points are missing from the figure. As expected, the shear across the interface, $\Delta \Omega_{1}-\Delta \Omega_{2}$, increases with $\Delta \Omega$, but it also displays a significant variation with $\Omega$. This variation has not previously been reported, and seems to be due to two opposing effects which occur as $\Omega$ is increased with $\Delta \Omega$ held constant. On the one hand, there is an increase in the interface slope, which in accordance with the gradient wind balance equation, will tend to increase the shear. On the other hand, the sidewall boundary-layer widths shrink, increasing the drag torque on both layers and tending to decrease the shear. These two competing effects evidently allow the shear to first increase, and then decrease, as $\Omega$ is increased with $\Delta \Omega$ held constant.

For each of the points plotted in figure $4(a)$, we calculate the Richardson number using (3.2). The results of this calculation at mid-radius, $r=9.4 \mathrm{~cm}$, are shown in figure $4(b)$. As $\Omega$ is increased with $\Delta \Omega$ held constant, we encounter Richardson numbers first greater than the critical values required for Kelvin-Helmholtz instability, then less than, and then greater than again. This is what we observed during the transitions to and from the $A X$ and $K H$ regimes in the experiments of $\S 2$, with the onset of Kelvin-Helmholtz instability being followed by a re-stabilization. 

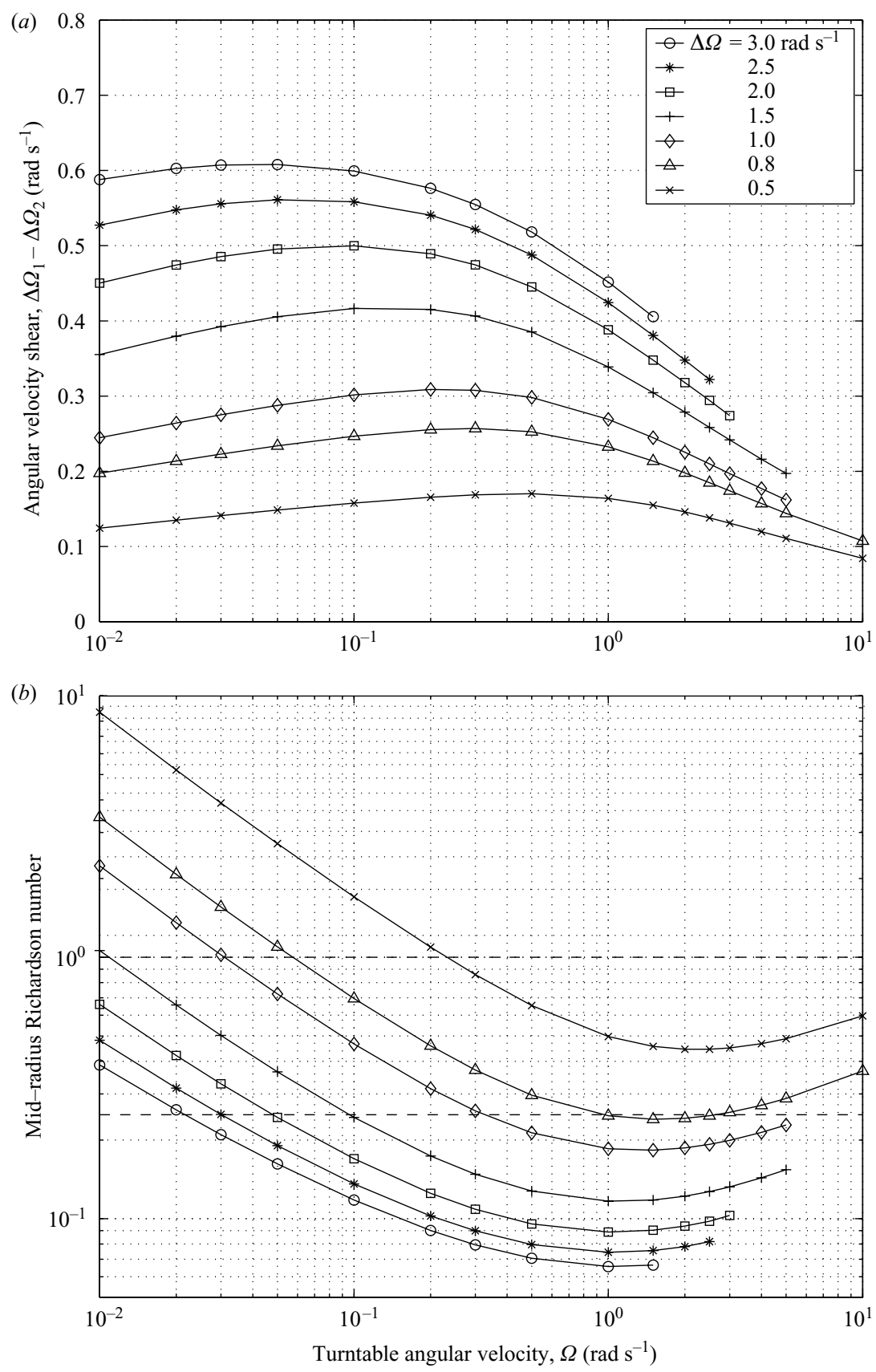

FIGURE 4. (a) Variation of angular velocity difference across the fluid interface, $\Delta \Omega_{1}-\Delta \Omega_{2}$, with turntable angular velocity, $\Omega$, for various fixed values of the differential lid rotation $\Delta \Omega$. (b) Variation of mid-radius Richardson number averaged over the interfacial Ekman layers, with turntable angular velocity, derived from the curves in $(a)$. Dashed lines corresponding to critical Richardson numbers of $1 / 4$ and 1 are also shown.

To further test the ability of this semi-analytical model to predict Kelvin-Helmholtz instability in the laboratory, we evaluate the dissipation parameter, $d$, and Froude number, $F$, at each of the points in figure $4(b)$, and thereby plot the Richardson 


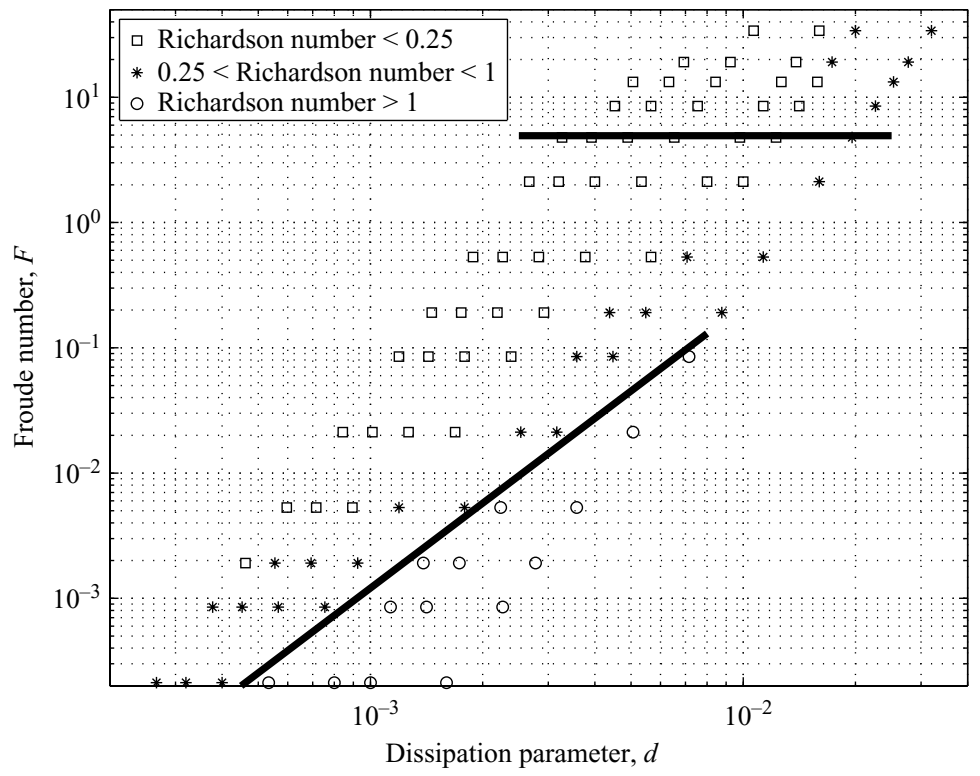

FIGURE 5. Model regime diagram showing the predicted regions of baroclinic and KelvinHelmholtz stability and instability in the $(d, F)$-plane, using the same axis limits and scales as in the corresponding laboratory regime diagram (figure 3). Kelvin-Helmholtz instability is expected wherever the Richardson number drops below a critical value, which is shown in this plot as 0.25 and 1 . The line of theoretical marginal baroclinic instability $\left(F_{\text {critical }}=\pi^{2} / 2\right)$ is over-plotted, together with the inferred location of the curve of marginal Kelvin-Helmholtz instability $\left(R i_{\text {critical }}=1\right)$.

number, $R i$, in $(d, F)$-space. The result is shown in figure 5. The experimental $A X \rightarrow$ $K H$ transition curve of figure 3 is well-reproduced by this simple analysis, for $R i_{\text {critical }}=1$. The discrepancies between the laboratory and model curves, which are slight, are probably due to the neglect of interfacial tension in the model, which can be significant for waves of small horizontal scales, and to the replacement of the gradient Richardson number with a bulk average over the interfacial Ekman layers. The presence of the $A X$ regime at around $d=0.01, F=3$ in figure 3 is not wellmodelled, as even the $R i_{\text {critical }}=0.25$ boundary in figure 5 is far from and a different shape to the experimental regime boundary.

We may now interpret better the shapes of the $A X$ and $K H$ regions in figure 3. There is no initial $A X$ regime in experiments $7-16$ because the shear is so large that the system is Kelvin-Helmholtz unstable even at the start of these experiments. We note here that the $A X$ regime around $d=0.01, F=3$ is missing from the series of experiments with decreasing turntable rotation $(\$ 2.2)$. This suggests that this part of the regime diagram is hysteretic and therefore beyond the prediction of simple linear stability analyses.

\section{Unbalanced modes in the baroclinically unstable fluid}

We now identify the particular generation mechanism responsible for the short waves in the $M R W$ and $M I W$ regimes, i.e. those present in figures $2(c)$ and $2(d)$. The semi-analytical investigation presented in $\S 3$ is impossible in this case, because we no longer have a simple axisymmetric background flow, but instead, a large-scale wave which has grown to finite amplitude owing to baroclinic instability. For this reason, we employ a numerical two-layer annulus quasi-geostrophic model named QUAGMIRE 
(Williams, Haine \& Read 2004a) to simulate the vortical flow in the laboratory. We note that the formal limits of applicability of quasi-geostrophic theory may be reached in the present experiments, since the Rossby number and vortical wave aspect ratio (i.e. layer depth divided by wavelength) may both approach unity in the $M R W$ and $M I W$ regimes (figure 3). Nevertheless, we find reasonable agreement, in terms of wavenumber, amplitude and phase speed, between vortical modes in the laboratory and those simulated in the numerical model, indicating that the quasi-geostrophic assumptions remain valid outside their formal domain of applicability in this case.

In this section, we use the model interface height and velocity fields to compute five indicators of short-scale wave generation in the $M R W$ regime. Some of the indicators are predictors of short-scale waves due to the shear instability mechanism, and others due to the spontaneous-adjustment mechanism. By investigating which of the five indicators best predicts the spatial locations of the laboratory short-scale waves, we are able to infer which of the two generation mechanisms is responsible.

\subsection{Indicators of short-scale wave generation}

We begin by defining the five generation indicators which are to be calculated using the model data.

\subsubsection{Horizontal divergence, $\delta$}

To first order in the Rossby number, the velocity fields in any quasi-geostrophic model are horizontally non-divergent, permitting the introduction of a streamfunction. At higher order, however, there must be a small non-zero horizontal divergence, $\delta=\nabla_{h} \cdot \boldsymbol{u}$, in order to allow the interface height to slowly evolve. Since the short modes are associated with a large horizontal divergence, there is the possibility that this field could serve as a good indicator of their generation.

From mass conservation, $\boldsymbol{\nabla} \cdot \boldsymbol{u}=0$, for an incompressible fluid, we have

$$
\delta=-\frac{\partial w}{\partial z} .
$$

Integrating over the lower annulus layer, at the top and bottom of which the vertical velocities are $(\mathrm{D} / \mathrm{D} t) h$ and 0 , respectively, where $h$ is the lower-layer depth, gives

$$
\delta=-\frac{1}{h}\left(\frac{\partial}{\partial t}+\boldsymbol{u} \cdot \nabla\right) h,
$$

with a similar expression for the upper layer. The horizontal divergence is a general indicator of short-wave emission, i.e. it is not specific to either the shear or spontaneous-adjustment mechanisms.

\subsubsection{Local Richardson number, $R i$}

The Richardson number discussed in $\S 3$ is expected to be a good indicator of shortscale wave generation by the shear instability mechanism. The general definition of bulk Richardson number for the annulus is

$$
R i_{\mathrm{b}}=\frac{2 g^{\prime} \sqrt{\bar{v} / \Omega}}{(\Delta \boldsymbol{u})^{2}},
$$

where $(\Delta \boldsymbol{u})^{2}=\left(\Delta u_{r}\right)^{2}+\left(\Delta u_{\theta}\right)^{2}$ is the velocity shear across the interface. Equation (4.3) is a generalization of (3.2), which applies only to the axisymmetric equilibrium flow.

\subsubsection{Brown indicator, $\phi$, and turbulent energy dissipation rate, $\epsilon$}

A number of shear instability indicators have been developed as complementary alternatives to the Richardson number. Two of these are the Brown indicator, $\phi$, and 
turbulent energy dissipation rate, $\epsilon$, first studied by Roach (1970) as indicators of clear air turbulence (CAT) in the atmosphere. CAT occurs in cloudless conditions at altitudes of around $10 \mathrm{~km}$, and is due to short-scale Kelvin-Helmholtz billows. It is occasionally severe enough to lift aeroplane passengers from their seats and cause injury or death (Roach \& Bysouth 2002), and so there are important practical reasons for developing a reliable indicator.

Roach notes that, on the one hand, some dynamical processes tend to increase the vertical shear in horizontal velocity, e.g. thermal wind balance giving a tropospheric jetstream in the atmosphere, or the imposed differential lid rotation in the annulus. On the other hand, viscous energy dissipation due to short-scale waves tends to reduce the shear. Roach makes an assumption of approximate balance between these two competing effects on short time scales, leaving the shear (and Richardson number) constant.

Roach imagines a thought experiment in the atmosphere in which the dissipation effect is switched off, destroying the balance and allowing an increase in the shear. $\mathrm{He}$ argues that the rate at which the small-scale features were dissipating energy just before the switch-off, must be equal to the rate of energy increase of the system just after, which is analytically derivable from the dynamical equations by setting the viscosity to zero. Using this approach, he calculates an energy dissipation rate of

$$
\epsilon= \begin{cases}\frac{(\Delta \boldsymbol{u})^{2}}{24} \phi & (\phi>0), \\ 0 & (\phi<0),\end{cases}
$$

where

$$
\phi=-\frac{1}{R i}\left(\frac{\partial}{\partial t}+\boldsymbol{u} \cdot \nabla\right) R i .
$$

$\Delta \boldsymbol{u}$ is the vertial shear in horizontal velocity associated with the tropospheric jetstream, taken to be the shear across the interface in the annulus, and $R i$ is the Richardson number for the large-scale flow, defined for the annulus by (4.3). The indicator $\phi$ takes its name from Brown (1973), who derived an approximate form which was more practical for operational diagnosis of CAT, though in the present study we use the direct definition (4.5). In Brown's paper, both $\phi$ and $\epsilon$ are shown to be better indicators of CAT than $R i$, and so we might expect the same to be true for short-scale wave generation in the annulus.

\subsubsection{Lighthill/Ford radiation term, $R$}

Lighthill (1952) presented a theory for the generation of sound waves by large-scale motions in a three-dimensional compressible adiabatic gas. The governing equations for Lighthill's system are isomorphic to the non-rotating shallow-water equations, with a correspondence between acoustic and gravity modes, and so the problem of generation of pure gravity waves had also essentially been solved by Lighthill. Ford (1994) extended the theory to include rotation, and thereby derived an inertia-gravity wave radiation term. The generation mechanism in this case is an evolving vortical motion rather than a velocity shear.

Ford's derivation uses the $f$-plane inviscid barotropic shallow-water equations. Two equations, obtained by taking the curl and the divergence of the momentum equation, are combined to produce a single equation:

$$
\left(\frac{\partial^{2}}{\partial t^{2}}+f^{2}-g H \nabla^{2}\right) \frac{\partial h}{\partial t}=\frac{\partial}{\partial t} \nabla \cdot \boldsymbol{G}+f \boldsymbol{k} \cdot \nabla \times \boldsymbol{G}+\frac{g}{2} \frac{\partial}{\partial t} \nabla^{2} h^{2},
$$


where

$$
\boldsymbol{G}=\boldsymbol{u} \nabla \cdot(h \boldsymbol{u})+(h \boldsymbol{u} \cdot \nabla) \boldsymbol{u},
$$

$\boldsymbol{k}$ is the unit vertical vector and $H$ is the ambient layer depth. The left-hand side of (4.6) is the linear shallow-water inertia-gravity wave operator acting on $\partial h / \partial t$, which turns out to be a more convenient variable than $h$. The right-hand side contains all of the nonlinear terms, which we refer to collectively as the Lighthill/Ford radiation term, $R$. The linear normal modes of (4.6) are shallow-water inertia-gravity waves, for which the intrinsic angular frequency $\omega$ and total horizontal wave vector $K$ are related by the dispersion equation:

$$
\omega^{2}=f^{2}+g H K^{2} .
$$

The right-hand side of Ford's original equation is written explicitly in Cartesian coordinates, but we retain the vector form here as we would like to calculate $R$ in the cylindrical geometry of the annulus. Ford goes on to derive an approximate form for $R$ based on small Froude number, though Froude numbers are larger than unity for flows of interest in the present system, and so we use the unapproximated form given by (4.6) and (4.7). Ford et al. (2000) argue that inertia-gravity waves will be generated in any region for which $R$ is non-zero, and they name the generation spontaneous-adjustment emission. These equations hold for either layer of the rotating annulus system, so long as we replace $g$ with the reduced gravity.

We note that Ford's theory is based on the shallow-water equations, and so the expression for $R$ given by (4.6) is an indicator of shallow-water inertia-gravity wave emission by an evolving shallow-water large-scale mode. Though the large-scale modes in the present system can reasonably be classified as shallow, it appears that the short-scale modes cannot. Their typical wavelengths (around $20 \mathrm{~mm}$ ) are significantly smaller than the layer depth $(125 \mathrm{~mm})$, suggesting that the observed short waves are in the deep-water regime with a different dispersion relation from (4.8). Nevertheless, we assert that Ford's theory is still applicable to the current system, since whether or not inertia-gravity waves will be generated by a given large-scale spatio-temporal structure is not expected to depend upon whether they would propagate in deep or shallow water, if emitted. Furthermore, we are interested primarily in the spatial patterns of the radiation term, not in the quantitative values. Since an energy source for shallow-water inertia-gravity waves will also tend to be an energy source for deep-water inertia-gravity waves, the spatial patterns of a deep water version of the radiation term should coincide with the spatial patterns of the shallow-water term, even though quantitative agreement may not be reached.

A summary of the properties of the five short-scale wave indicators defined above is given in table 3, where the indicators are classified according to whether they are expected to predict generation due to the spontaneous adjustment or vertical shear instability mechanisms.

\subsection{Calculation of indicators using model data}

Figure 6 shows plots of the five indicators of short-scale wave generation, calculated from a numerical experiment with $\Delta \Omega=0.15 \mathrm{rad} \mathrm{s}^{-1}$ and $\Omega=3.00 \mathrm{rad} \mathrm{s}^{-1}$, for which the equilibrated flow is a wavenumber two mode of constant amplitude. The model interface height field is also shown in the figure, and the wave amplitude compares reasonably well with that in the laboratory. If we observed this interface height field in a laboratory experiment, we would expect short-scale wave generation at lowto mid-radii near $\theta=90^{\circ}$ ('12 o'clock') and $\theta=270^{\circ}$ ('6 o'clock'), as can be seen by comparison with figure $2(c)$. We are therefore interested in which (if any) of the five 
Indicator name

Horizontal divergence

Richardson number

Brown indicator

Energy dissipation rate

Lighthill/Ford radiation term
Definition

$$
\delta=\nabla_{h} \cdot \boldsymbol{u}
$$

$R i \propto 1 /(\Delta \boldsymbol{u})^{2}$

$\phi=-(\mathrm{D} / \mathrm{D} t) \ln R i$

$\epsilon \propto(\Delta \boldsymbol{u})^{2} \phi$

$R=$ nonlinear terms in (4.6)
Mechanism Generation condition

SA and SI

$\begin{array}{rlrl} & \text { SI } & R i & <1 \\ \text { SI } & \phi & >0 \\ \text { SI } & \epsilon & >0 \\ \text { SA } & |R| & >0\end{array}$

TABLE 3. Summary of the five short-scale wave indicators to be calculated using data from the numerical model. The particular generation mechanism associated with each indicator is listed (shear instability, $S I$, or spontaneous adjustment, $S A$ ) together with the condition which must be satisfied in order for generation to be expected.

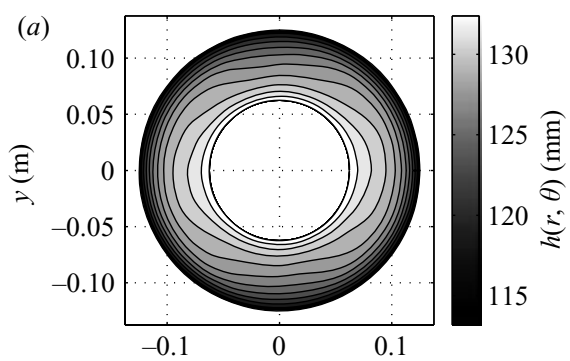

(b)

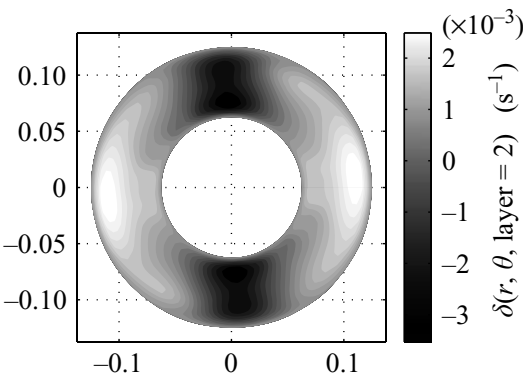

(c)

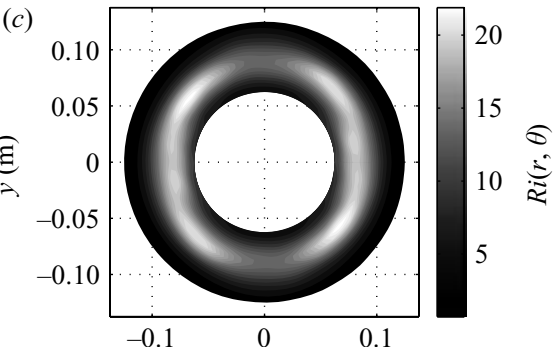

(e)

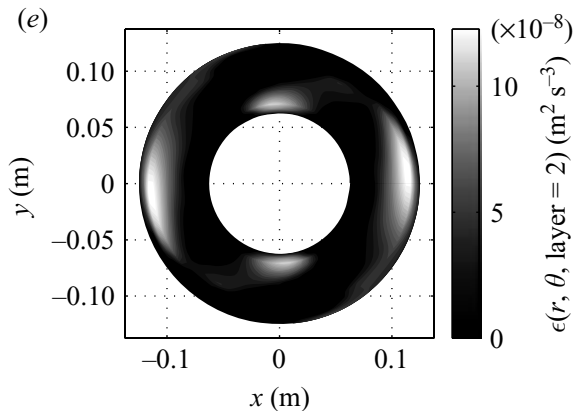

(d)

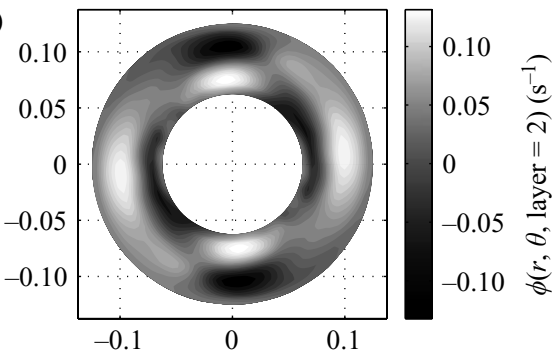

$(f)$

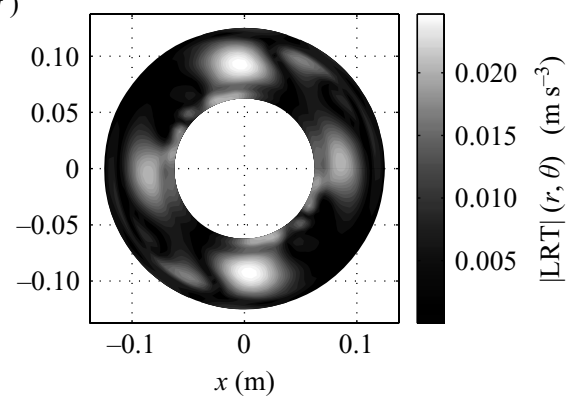

FIGURE 6. Plots of indicators of short-scale wave generation calculated from the QUAGMIRE model data, for a numerical experiment with $\Delta \Omega=0.15 \mathrm{rad} \mathrm{s}^{-1}$ and $\Omega=3.00 \mathrm{rad} \mathrm{s}^{-1}$. (a) Interface height, $(b)$ lower-layer horizontal velocity divergence, $(c)$ Richardson number, $(d)$ lowerlayer Brown indicator, $(e)$ lower-layer energy dissipation rate, and $(f)$ lower-layer Lighthill/ Ford radiation term magnitude. The corresponding plots for the upper layer (not shown) are very similar to these. 
indicators would predict short-scale wave generation at these (and only these) angular positions, based on the criteria in table 3 .

The horizontal divergence indicator shows four large-amplitude regions, but this includes two at which short-scale waves are not observed in the laboratory. The Richardson number shows local minima with respect to azimuth at the two expected regions, but is at its smallest close to the outer sidewall which is not a laboratory generation region. The Brown indicator has large positive maxima exactly where the short laboratory waves appear, but there are two equally large maxima elsewhere in the annulus. Similarly, the energy dissipation rate has two maxima too many to be a reliable indicator, and both of the unwanted maxima are larger than the maxima in the expected locations. The Lighthill/Ford radiation term has large global maxima at the two expected regions, and two weaker local maxima at other locations in the annulus.

The three vertical shear instability indicators have each over-predicted regions of short-scale wave generation. The Lighthill/Ford expression, an indicator of spontaneous-adjustment emission, gives the best fit with the laboratory observations. It has large values exactly where the short waves appear in the laboratory. It is important to note that a parallax error is present in figure 2, but not in figure 6 . Simple geometry reveals that the inner cylinder blocks approximately the innermost $5 \mathrm{~mm}$ of the interface from the line of sight of the video camera. The short-scale waves in figure 2(c) are therefore further from the inner cylinder than they seem, and the agreement between the laboratory image of figure $2(c)$ and the model Lighthill/Ford plot of figure 6 is better than it initially appears. There are smaller subsidiary local maxima in the Lighthill/Ford plot, but the values taken there may not be large enough for incipient laboratory short waves to overcome the effects of viscous dissipation not included in the Lighthill/Ford theory - and grow to an amplitude which would make them visible. We conclude that spontaneous-adjustment emission is likely to be responsible for the $M R W$ regime short-scale wave generation in the laboratory. The same conclusion is reached when the five indicators are plotted for azimuthal wavenumber 1 and 3 flows (not shown).

It is instructive to break down the Lighthill/Ford indicator in (4.6) into its three constituent nonlinear terms, which are individually plotted in figure 7 . As might be expected for a quasi-geostrophic flow, the two time derivative terms, $\partial(\nabla \cdot G) / \partial t$ and $0.5 g \nabla^{2}\left(\partial h^{2} / \partial t\right)$, are each much smaller than the remaining, non-time derivative term, $f \boldsymbol{k} \cdot \boldsymbol{\nabla} \times \boldsymbol{G}$. A simple scale analysis shows that the factor by which they differ is of the same order as the bulk Rossby number, which is $1 / 40$ in this case. It therefore seems that, for many practical purposes, it may be a good approximation to retain only the middle term on the right-hand side of (4.6).

As regards the baroclinically stable flow regime, $\delta, \phi$ and $\epsilon$ are each identically zero since the flow is then steady and axisymmetric. Most of the contributions to $R$ are also zero, and those which are not zero are very small due to the heavy differentiation, giving a value for $R$ which is around $10^{6}$ times smaller than in figure $6(f)$ (not shown). Only $R i$ is non-negligible, reinforcing the conclusions of $\S 3$ that the laboratory short waves in the baroclinically stable regime are generated by a shear instability.

As noted above, a large value of the Lighthill/Ford term is simply an indication of a tendency for the fluid to generate inertia-gravity waves. Whether they will actually appear depends upon whether the rate of energy input into the inertia-gravity modes, due to the loss of energy of the adjusting large-scale flow, exceeds the rate at which energy in the short-scale modes can be dissipated. To illustrate this, we recall our preliminary series of laboratory experiments briefly referred to in $\S 2.2$, in which the 

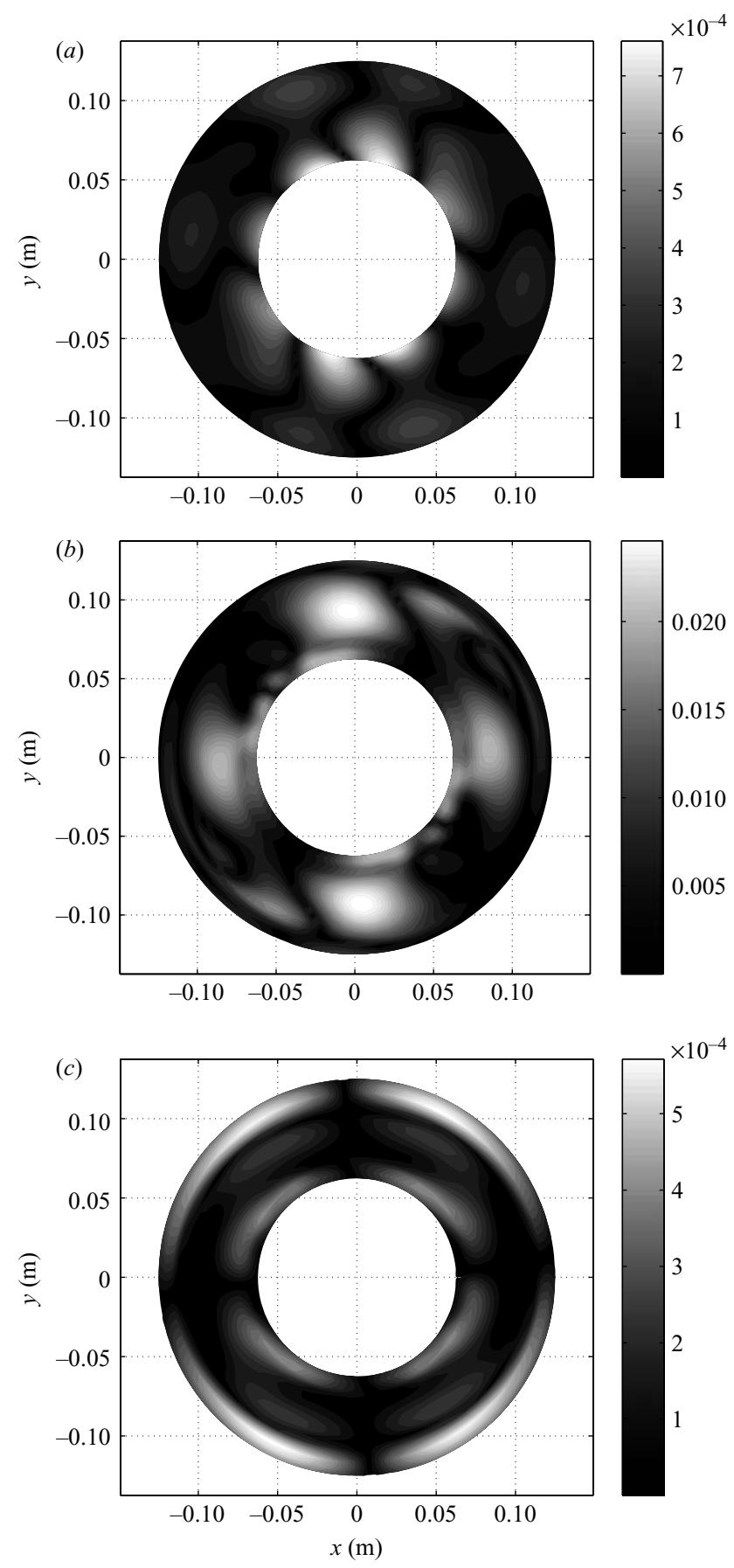

FIGURE 7. Plots of the magnitudes of the three contributions to the Lighthill/Ford radiation indicator shown in figure $6(f)$, i.e. the three nonlinear terms on the right-hand side of (4.6): (a) $\partial(\nabla \cdot \boldsymbol{G}) / \partial t$, (b) $f \boldsymbol{k} \cdot \nabla \times \boldsymbol{G}$ and $(c) 0.5 g \nabla^{2}\left(\partial h^{2} / \partial t\right)$. Note the changes in the colourbar scales between the plots. The units are $\mathrm{ms}^{-3}$. 
surfactant which was added to the working liquids for the experiments described herein was not added. This meant that the dissipative effects of interfacial tension remained large, and we found that the generation of inertia-gravity waves in the baroclinically unstable regime was suppressed (Williams et al. 2004a).

The wavelength and wake angle of the inertia-gravity waves in the baroclinically unstable regime can be estimated using a simple analytical model. We use the ship wave problem studied by Lighthill (1978), in which an object moving in a straight line at speed $V$ in a fluid generates a wake of deep-water surface gravity waves. It is shown in that analysis, using geometrical arguments based on the phase speed being twice the group speed, that waves at the edge of the wake have a wavelength of $\lambda=(4 \pi / 3)$ $\left(V^{2} / g\right)$, and that their crests meet each other at an angle of $\alpha=90^{\circ}+\sin ^{-1}(1 / 3) \approx 109^{\circ}$.

This suggests a model for the $M R W$ and $M I W$ short-scale wave generation in the laboratory experiments, in which the entire large-scale wave is taken to be the extended moving object which causes the inertia-gravity wave generation. The Lighthill theory is non-rotating, but as in $\S 3$ we assume that the laboratory short-scale waves are not strongly influenced by rotation. In figure $2(c)$, the short-scale wave crest angle is around $90^{\circ}$, reasonably close to the predicted angle. In the same figure, the measured angular drift speed of the large-scale wave is $0.11 \mathrm{rad} \mathrm{s}^{-1}$, giving a linear velocity at mid-radius, $r=9.4 \mathrm{~cm}$, of $V=1.0 \mathrm{~cm} \mathrm{~s}^{-1}$. With this velocity, and using the reduced gravity in place of $g$, the Lighthill theory predicts $\lambda=8 \mathrm{~mm}$, which is in reasonable agreement with the observed wavelength given Lighthill's assumptions of no rotation and a point generating object.

\section{Conclusions}

We have reported on the results of a laboratory investigation using a rotating twolayer annulus system, in which both vortical and divergent eigenmodes are excited. We have described a series of experiments in which the Froude number varies by five orders of magnitude, the Rossby number by three orders of magnitude, and the dissipation parameter by two orders of magnitude, and we have categorized the flow according to the existence of divergent and/or vortical modes. Vortical modes, manifest as baroclinic instability waves, are exhibited whenever the system parameters are such that the Froude number is supercritical (i.e. $F>F_{\text {critical }} \equiv \pi^{2} / 2$ ), as expected. The principal contribution of our study is to have determined the circumstances in which divergent modes are excited in the annulus, and to have inferred the mechanisms by which they are generated.

We used a semi-analytical approach to compute the Richardson number at a wide range of points in parameter space, assuming no baroclinic instability. As the Coriolis parameter is increased from zero, this algorithm generally predicts a Richardson number which is at first supercritical (no Kelvin-Helmholtz instability), then subcritical (Kelvin-Helmholtz instability), and then supercritical again. This behaviour is due to increases in both the lateral boundary drag forces and the interface slope as the background rotation is increased, resulting in variations in the velocity shear across the fluid interface. The predictions of this approach are reproduced in the laboratory, with the experimental regime diagram showing a window of short-wave generation bounded by regions of no short-wave generation, though the location in parameter space of one edge of the window is not well modelled. Divergent modes, manifest as Kelvin-Helmholtz instability waves, are therefore exhibited whenever the system parameters are such that the Richardson number is subcritical (i.e. $R i<R i_{\text {critical }} \equiv 1$ ), as expected. 
Divergent modes are also seen in spatially confined regions in the baroclinically unstable fluid. Their chevron-shaped structure closely resembles that of the inertiagravity waves generated by geostrophic adjustment of the tropospheric jetstream, in the high-resolution numerical simulations of O'Sullivan \& Dunkerton (1995). In this regime, we used a quasi-geostrophic numerical model to investigate the incipient generation of divergent modes by the large-scale mode, according to two distinct mechanisms. In contrast with the baroclinically stable case, the model Richardson number is now not subcritical, making it unlikely that these short modes are generated by a local shear instability. This is in accord with the development of vortical eddies owing to a baroclinic instability being accompanied by the development of a baroclinic mean flow correction, which acts in such a sense as to reduce the background velocity shear (e.g. figure 3 of Lovegrove, Moroz \& Read 2001). The model Lighthill/Ford radiation indicator has maxima at exactly those spatial locations at which we observe short modes in the laboratory. Divergent modes, manifest as freely-propagating spontaneously-emitted inertia-gravity waves, are therefore locally exhibited wherever this indicator is large in the baroclinically unstable regime. In additional laboratory experiments with an increased interfacial tension, the generation of inertia-gravity waves is significantly suppressed owing to enhanced dissipation. This would suggest that a large Lighthill/Ford indicator is a necessary, but not sufficient condition, for the appearance of the short waves. Future work will extend Ford's theory to include dissipative effects, with the goal of obtaining an improved radiation indicator.

Further work is required to determine in more detail how to properly interpret the Lighthill/Ford indicator. For example, Benney (1977) has presented a general theory for interactions between long and short waves. In the present context, his results would permit an exchange of energy (owing to a resonant triad interaction) from vortical to inertia-gravity waves, if and only if the phase speed of the vortical mode were equal to the group speed of the inertia-gravity wave. It is unclear whether this implies that only inertia-gravity waves of a certain wavelength can be generated by the Lighthill/Ford source term. More recently, Zhang (2004) has found the residual of the nonlinear balance equation to be a useful index for predicting gravity wave generation. Further research is required to identify and explore the relationships between the Lighthill/Ford indicator, the nonlinear balance equation residual, and the Benney resonance criterion.

The regimes which occur in the atmosphere and the baroclinically unstable annulus, as defined by the Rossby, Froude and Richardson numbers, are reasonably similar. Therefore, the Lighthill/Ford radiation term may function as a good predictor of spontaneous-adjustment emission in the atmosphere, as it does in the laboratory. If further work reveals this to be true, it may have the potential to improve parameterizations of gravity wave drag in general circulation models. For example, it would raise the attractive prospect of calculating the radiation term at each time step, and activating the parameterization only in those regions where the term is larger than some specified threshold. If the calculation of the radiation indicator were performed using only the non-time derivative term, shown in $\S 4$ to be the largest in the present context, then the extra processing time required should be small. The results of this study clearly give a strong motivation for more fully investigating the geophysical relevance and applicability of the Lighthill/Ford indicator.

P. D. W. acknowledges financial support under a research studentship from the UK Natural Environment Research Council, held at the University of Oxford, with award 
reference number GT04/1999/AS/0203. The authors thank three anonymous referees for their helpful comments.

\section{REFERENCES}

ACheson, D. J. 1990 Elementary Fluid Dynamics. Oxford University Press.

AfanAsyev, Y. 2003 Spontaneous emission of gravity waves by interacting vortex dipoles in a stratified fluid: laboratory experiments. Geophys. Astrophys. Fluid Dyn. 97, 79-95.

BenNEY, D. J. 1977 A general theory for interactions between short and long waves. Stud. Appl. Maths 56, 81-94.

Berman, A. S., Bradford, J. \& Lundgren, T. S. 1978 Two-fluid spin-up in a centrifuge. J. Fluid Mech. 84, 411-431.

Bradford, J., Berman, A. S. \& Lundgren, T. S. 1981 Nongeostrophic baroclinic instability in a two-layer rotating system. J. Atmos. Sci. 38, 1376-1389.

Brown, R. 1973 New indices to locate clear-air turbulence. Met. Mag. 102, 347-361.

Chandrasekhar, S. 1961 Hydrodynamic and Hydromagnetic Stability. Oxford University Press.

Dalin, P., Kirkwood, S., Moström, A., Stebel, K., Hoffmann, P. \& Singer, W. 2004 A case study of gravity waves in noctilucent clouds. Ann. Geophys. 22, 1875-1884.

ECKeRmann, S. D. \& Vincent, R. A. 1993 VHF radar observations of gravity-wave production by cold fronts over southern Australia. J. Atmos. Sci. 50, 785-806.

Ekman, V. W. 1905 On the influence of the earth's rotation on ocean currents. Ark. Math. Astr. Fys. 2, $1-52$.

FoRD, R. 1994 Gravity wave radiation from vortex trains in rotating shallow water. J. Fluid Mech. 281, 81-118.

Ford, R., McIntyre, M. E. \& Norton, W. A. 2000 Balance and the slow quasimanifold: some explicit results. J. Atmos. Sci. 57, 1236-1254.

FritTs, D. C. 1982 Shear excitation of atmospheric gravity waves. J. Atmos. Sci. 39, 1936-1952.

FRITTS, D. C. 1984 Shear excitation of atmospheric gravity waves. Part II: Nonlinear radiation from a free shear layer. J. Atmos. Sci. 41, 524-537.

FritTs, D. C. \& LuO, Z. 1992 Gravity wave excitation by geostrophic adjustment of the jet stream. Part I: Two-dimensional forcing. J. Atmos. Sci. 49, 681-697.

Griffiths, R. W. \& Linden, P. F. 1981 The stability of buoyancy-driven coastal currents. Dyn. Atmos. Oceans, 5, 281-306.

Hart, J. E. 1972 A laboratory study of baroclinic instability. Geophys. Fluid Dyn. 3, 181-209.

HART, J. E. 1985 A laboratory study of baroclinic chaos on the f-plane. Tellus 37A, 286-296.

HaRT, J. E. \& KitTELman, S. 1986 A method for measuring interfacial wave fields in the laboratory. Geophys. Astrophys. Fluid Dyn. 36, 179-185.

Hines, C. O. 1988 Tropopausal mountain waves over Arecibo: a case study. J. Atmos. Sci. 46, 476-488.

Hough, S. S. 1898 On the application of harmonic analysis to the dynamical theory of the tides. II: On the general integration of Laplace's dynamical equations. Phil. Trans. R. Soc. Lond. 191, 139-185.

James, I. N. 1977 Stability of Flow in a Slowly Rotating Two-Layer System. Occasional Note Met O 21/77/2. Geophysical Fluid Dynamics Laboratory, UK Meteorological Office.

KING, J. C. 1979 An experimental study of baroclinic wave interactions in a two-layer system. Geophys. Astrophys. Fluid Dyn. 13, 153-167.

KLeIN, P. 1990 Transition to chaos in unstable baroclinic systems: a review. Fluid Dyn. Res. 5, 235-254.

Lighthill, M. J. 1952 On sound generated aerodynamically. I: General theory. Proc. R. Soc. Lond. 211, 564-587.

Lighthill, M. J. 1978 Waves in Fluids. Cambridge University Press.

Longuet-Higgins, M. 1968 The eigenfunctions of Laplace's tidal equations over a sphere. Phil. Trans. R. Soc. Lond. 262 (1132), 511-607.

Lovegrove, A. F., Read, P. L. \& Richards, C. J. 1999 Generation of inertia-gravity waves by a time-dependent baroclinic wave in the laboratory. Phys. Chem. Earth (B) 24, 455-460. 
Lovegrove, A. F., Read, P. L. \& Richards, C. J. 2000 Generation of inertia-gravity waves in a baroclinically unstable fluid. Q. J. R. Met. Soc. 126, 3233-3254.

Lovegrove, A. F., Moroz, I. M. \& Read, P. L. 2001 Bifurcations and instabilities in rotating two-layer fluids: I. f-plane. Nonlinear Proc. Geophys. 8, 21-36.

Luo, Z. \& FRITTS, D. C. 1993 Gravity wave excitation by geostrophic adjustment of the jet stream. Part II: Three-dimensional forcing. J. Atmos. Sci. 50, 104-115.

Margules, M. 1893 Luftbewegungen in einer rotierenden Sphäroidschale. Sitzungsberichte der Kaiserliche Akad. Wiss. Wien, Part IIA, 102, 11-56.

O’Sullivan, D. \& Dunkerton, T. J. 1995 Generation of inertia-gravity waves in a simulated life cycle of baroclinic instability. J. Atmos. Sci. 52, 3695-3716.

Pedlosky, J. 1987 Geophysical Fluid Dynamics. Springer.

READ, P. L. $1992 a$ Applications of singular systems analysis to 'baroclinic chaos'. Physica D 58, 455-468.

READ, P. L. $1992 b$ Dynamics and instabilities of Ekman and Stewartson layers. Chapter III.1 in Rotating Fluids in Geophysical and Industrial Applications. Springer.

RoAch, W. T. 1970 On the influence of synoptic development on the production of high level turbulence. Q. J. R. Met. Soc. 96, 413-429.

Roach, W. T. \& Bysouth, C. E. 2002 How often does severe clear air turbulence occur over tropical oceans? Weather 57, 8-19.

Rossby, C. G. 1938 On the mutual adjustment of pressure and velocity distributions in certain simple current systems. II. J. Mar. Res. 2, 239-263.

SATO, K. 1994 A statistical study of the structure, saturation and sources of inertio-gravity waves in the lower stratosphere observed with the MU radar. J. Atmos. Terr. Phys. 56, 755-774.

Stewartson, K. 1957 On almost rigid rotations. J. Fluid Mech. 3, 17-26.

Williams, P. D., Read, P. L. \& Haine, T. W. N. 2003 Spontaneous generation and impact of inertia-gravity waves in a stratified, two-layer shear flow. Geophys. Res. Lett. 30, 2255, DOI:10.1029/2003GL018498.

Williams, P. D., Haine, T. W. N. \& Read, P. L. $2004 a$ Stochastic resonance in a nonlinear model of a rotating, stratified shear flow, with a simple stochastic inertia-gravity wave parameterization. Nonlinear Proc. Geophys. 11, 127-135.

Williams, P. D., Read, P. L. \& Haine, T. W. N. $2004 b$ A calibrated, non-invasive method for measuring the internal interface height field at high resolution in the rotating, two-layer annulus. Geophys. Astrophys. Fluid Dyn. 98, 453-471.

ZhANG, F. 2004 Generation of mesoscale gravity waves in upper-tropospheric jet-front systems. J. Atmos. Sci. 61, 440-457. 